\title{
NEGATIVE ION PHOTOELECTRON SPECTROSCOPY OF $\mathbf{P}_{\mathbf{2}}^{-}$
}

\author{
J.T. SNODGRASS, J.V. COE, C.B. FREIDHOFF, \\ K.M. McHUGH and K.H. BOWEN \\ Deparment of Chemisny, The Johns Hopkins Universiny, Baltimore, MD 2I2J8, USA
}

Received 16 September 1985

\begin{abstract}
We have recorded the photoelectron spectrum of $\mathrm{P}_{2}^{-}$using a Penning ion source and a negative ion photoclectron spectrometer. The adiabatic electron allinity of $P_{2}$ is determined to be $0.589 \pm 0.025 \mathrm{eV}$. The negative ion parameters determined in this work are: $\omega_{c}^{\prime \prime}\left(P_{\underline{2}}^{-}\right)=640 \pm 50 \mathrm{~cm}^{-1}, R_{c}^{\prime \prime}\left(P_{\underline{2}}^{-}\right)=1.979 \pm 0.010 \dot{A}$. and $D_{0}\left(P_{\underline{2}}^{-}\right)=4.88 \pm 0.06 \mathrm{eV}$.
\end{abstract}

\section{Introduction}

Extensive spectroscopic data is available about the $P_{2}$ molecule [1]. By contrast relatively little information is available about its negative ion, $P_{2}^{-}$. This ion appears to have first bcen observed mass spectrometrically by Duke]'skii and Zanberg [2] in 1952. Carette and Kerwin [3] generated $\mathrm{P}_{\mathbf{2}}^{-}$by dissociative electron attachment to $\mathbf{P}_{4}$ in 1961 . From their work they were able to give an estimate of $0.3 \pm 0.5 \mathrm{eV}$ for the electron affinity of $P_{\mathbf{2}}$. Later, the appearance potential and ion translational energy measurements of Bennett, Margrave and Franklin [4] gave an electron affinity for $\mathrm{P}_{2}$ of $0.24 \pm 0.23 \mathrm{eV}$. A low-resolution, tunablefrequency threshold photodetachment experiment on $\mathbf{P}_{\mathbf{2}}^{-}$was conducted by Feldmann, Rackwitz, Kaiser and Heinicke [5] using a Penning source to generate $\mathbf{P}_{\mathbf{2}}^{-}$. They observed a broad rise in the photodetachment cross section with little discernible structure. Upon assuming that vibrationally excited $\mathrm{P}_{2}^{-}$ions were not present, they were able to provide an upper limit to the electron affinity of $P_{2}$ of $0.65 \mathrm{eV}$ from the extrapolated threshold behavior of their spectrum. Here, we report the recording of the photodetachment spectrum of $\mathrm{P}_{\mathbf{2}}^{-}$by negative ion photoelectron spectroscopy. We obtain a highly structured photoelectron spectrum for $\mathrm{P}_{\mathbf{2}}^{-}$from which the adiabatic electron affinity of $P_{2}$ and the spectroscopic constants $B_{\mathrm{e}}^{\prime \prime}$ and $\omega_{\mathrm{e}}^{\prime \prime}$ for the $\mathrm{P}_{2}$ ion are determined. Using these data, we also calculate $R_{r}^{\prime \prime}$ and $D_{0}$ for $\mathrm{P}_{2}^{-}$.

\section{Experimental}

In negative ion photoelectron spectroscopy a massselected negative ion beam is crossed with a îxed-frequency laser beam, and the resulting photodetached electrons are subjected to energy analysis. Our apparatus, which has been described previously [6], employs a Wien velocity filter for mass selection, an argon ion laser operated intra-cavity in the ion-photon interaction region, and a magnetically shielded, hemispherical electron energy analyzer. The ion source used in this work was a cold-cathode Penning discharge source of the type originally developed by Heinicke et al. [7]. This source consists of a cylindrical anode, two disc cathodes located near opposite ends of the anode, an oven for supplying vapors to the anode, and an electromagnet for generating a magnetic field along the axis of the anode cylinder. Negative ions were extracted radially through an aperture in the side of the anode into the photoelectron spectrometer.

To generate $\mathbf{P}_{\mathbf{2}}-$ and other phosphorus-containing negative ions, red phosphorus was heated in the oven to $\approx 325^{\circ} \mathrm{C}$ to supply phosphorus vapor to the anode, and argon was added as the discharge support gas (nominal pressure $\approx 0.3$ Torr). Mass spectra of the negative ions produced in this fashion showed the presence of $\mathrm{P}_{n=1-9}^{-}, \mathrm{PO}^{-}, \mathrm{PO}_{2}^{-}, \mathrm{PO}_{3}^{-}, \mathrm{PH}^{-}$, and $\mathrm{PH}_{2}^{-}$. Several of these species are shown in the negative ion mass spectrum presented in fig. 1. 


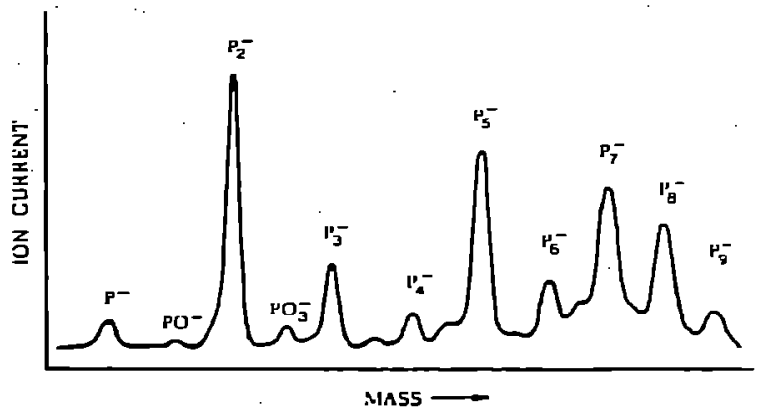

Fig. 1. A negative ion mass spectrum showing some of the phosphoruscontaining species generated by the Penning ion source during these experiments.

\section{Results and discussion}

\subsection{Data for $P_{\overline{2}}$}

Our photoelectron spectrum of $\mathrm{P}_{\mathbf{2}}^{-}$, which was recorded with $2.540 \mathrm{eV}$ photons, is presented in fig. 2 . The well-known atomic photodetachment transitions [8] of $\mathrm{P}^{-}$were used to calibratc our $\mathrm{P}_{2}^{-}$spcetra and to provide an energy scale compression factor. In order to obtain peak centers and peak heights of optimal accuracy, the raw data peaks were fit to an asymmetric Gaussian function prior to analysis.

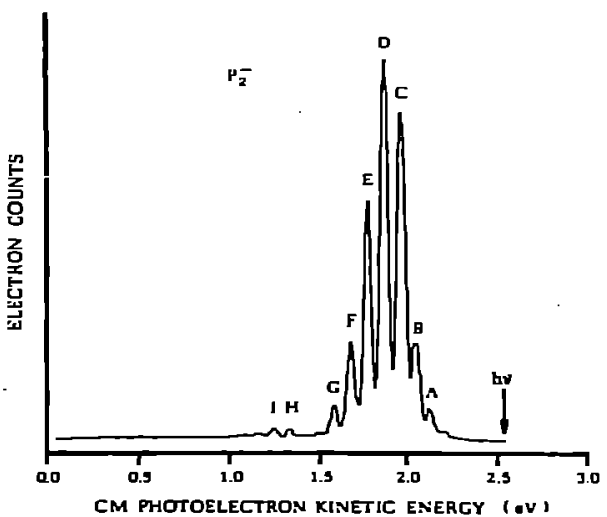

Fig. 2. The photoelectron spectrum of $\mathbf{P}_{2}^{-}$presented in terms of center-of-mass electron kinetic energies. This spectrum was recorded over about $1 / 2 \mathrm{~h}$ with $0.8 \times 10^{-9} \mathrm{~A}$ of $\mathrm{P}_{2}^{-}$ion current and $150 \mathrm{~W}$ of $2.540 \mathrm{eV}$ photons.
Table 1

Peak locations, spacing, and assignments of the $\mathbf{P}_{\mathbf{2}}^{-}$photoelectron spectrum

\begin{tabular}{llll}
\hline Peak & $\begin{array}{l}\text { C.m. electron } \\
\text { kinetic energy } \\
(\mathrm{eV})\end{array}$ & $\begin{array}{l}\text { Adjacent } \\
\text { spacing } \\
\left(\mathrm{cm}^{-1}\right)\end{array}$ & $\begin{array}{l}\text { Assignment } \\
\left(v^{\prime}, v^{\prime \prime}\right) \\
\mathbf{P}_{2}{ }^{\prime \prime} \Sigma_{\mathrm{g}}^{+}\end{array}$ \\
\hline A & 2.110 & $\mathrm{P}_{2}^{-},{ }^{2} \Pi_{\mathrm{g}}$ \\
B & 2.032 & 630 & $(0,2)$ \\
C & 1.954 & 630 & $(0,1)$ \\
D & 1.856 & 790 & $(0,0)$ \\
E & 1.758 & 790 & $(1,0)$ \\
F & 1.660 & 790 & $(2,0)$ \\
G & 1.564 & 770 & $(3,0)$ \\
\hline
\end{tabular}

\subsection{Spectral assignment}

Table 1 presents the center-of-mass electron kinetic energies of the peak centers for peaks $A$ through $G$ as well as the adjacent peak spacings and our assignments. The spacing between peak couples $C / D, D / E, E / F$ and $\mathrm{F} / \mathrm{G}$ are all approximately equal to the vibrational level spacing in the $\mathrm{X}^{1} \Sigma_{\mathrm{g}}^{+}$state of $\mathrm{P}_{2}\left(\Delta G_{1 / 2}=775\right.$ $\mathrm{cm}^{-1}$ ). The spacings between peak couples $A / B$ and $\mathrm{B} / \mathrm{C}$, however, are smaller and are equal to $630 \mathrm{~cm}^{-1}$. We assign peaks, C, D, E, F and G, respectively, as transitions from the $v^{\prime \prime}=0, \mathrm{X}^{2} \Pi_{\mathrm{g}}$ state of $\mathrm{P}_{2}^{-}$to the $v^{\prime}=0,1,2,3$ and 4 vibrational levels in the $X^{1} \Sigma_{g}^{+}$ state of $\mathbf{P}_{2}$. We assign peaks $A$ and $B$, respectively, to transitions from the $v^{\prime \prime}=2$ and the $v^{\prime \prime}=1$ vibrational levels of the $\mathrm{X}^{2} \Pi_{\mathrm{g}}$ state of $\mathrm{P}_{2}^{-}$to the $v^{\prime}=0, \mathrm{X}^{1} \Sigma_{\mathrm{g}}^{+}$ state of $P_{2}$. This assignment of peaks $C$ through $G$ to transitions originating in the vibrational ground state of $\mathrm{P}_{2}^{-}$, and of peaks $\mathrm{A}$ and $\mathrm{B}$ to transitions originating in vibrationally excited states of $P_{2}^{-}$is supported by the observation that while the relative intensities of peaks $C$ through $G$ are invariant with ion source conditions, the intensities of peaks $A$ and $B$ relative to peaks $\mathrm{C}$ through $\mathrm{G}$ change considerably with ion source conditions.

In order to confirm these assignments and to obtain spectroscopic parameters for $\mathrm{P}_{2}^{-}$, a FranckCondon analysis and a comparative modeling of the 
photoelectron spectrum was performed. When the spectrum was modeled on the assumption that peak $C$ is the $\left(v^{\prime}=1, v^{\prime \prime}=0\right)$ transition, the peak predicted to occur in the vicinity of peak $B$ is found to be taller and at higher electron kinetic energy than it is actually observed to be. When, however, the spectrum is modeled on the assumption that peak $C$ is the $(0,0)$ transition, good agreement is obtained with the observed spectrum. Negative ion potential parameters for $\mathrm{P}_{\mathbf{2}}^{-}$obtained from the spectrum and the modeling process are $\omega_{\mathrm{e}}^{\prime \prime}=640 \pm 50 \mathrm{~cm}^{-1}, B_{\mathrm{c}}^{\prime \prime}=0.2776 \pm 0.0028$ $\mathrm{cm}^{-1}$, and therefore $R_{\mathrm{e}}^{\prime \prime}=1.979 \pm 0.010 \mathrm{~A}$. The longer $R_{\mathrm{e}}$ and the lower $\omega_{\mathrm{e}}$ which we find for $\mathrm{P}_{2}^{-}$as compared to $\mathrm{P}_{2}$ are expected because of the antibonding character of the excess electron in $\mathbf{P}_{\mathbf{2}}^{-}$.

The relatively small peaks, $H$ and 1 , as well as other low signal peaks which we occasionally observe at still lower electron kinetic energies, do not appear to be $\mathrm{P}_{\mathbf{2}}^{-}$transitions. $\mathrm{P}_{2}$ does not possess low-lying electronic states that could account for any of these peaks [1]. These features are probably due to the photodetachment of "impurity" ions with masses close to that of $\mathrm{P}_{2}^{-}$which have "leaked" into the ionphoton interaction region at our available mass resolution. The $\mathrm{PO}_{2}^{-}$ion seems an unlikely possibility as an interferent ion because the electron affinity of $\mathrm{PO}_{2}$ is known to be greater than $3 \mathrm{eV}[9,10]$. The $\mathrm{P}_{2} \mathrm{H}^{-}$, $\mathrm{P}_{2} \mathrm{H}_{2}^{-}$and $\mathrm{P}_{2} \mathrm{H}_{3}^{-}$ions, on the other hand, have been observed previously in Penning sources [11] and are probably more reasonable candidates.

\subsection{Ion vibrational temperatures}

In an effort to investigate the vibrational temperature of molecular negative ions produced in Penning sources, we recorded photoelectron spectra of $\mathrm{P}_{\mathbf{2}}^{-}$and $\mathrm{O}_{2}^{-}$under a varicty of Penning source operating conditions. (The photoelectron spectrum of $\mathrm{O}_{2}^{-}$, generated in a hot-cathode ion (Branscomb) source, is well understood [12].) Rough temperatures for the various hot-band peaks were calculated from the Boltzmann distribution law by comparing the intensities of individual hot-band peaks with the intensity of their corre. sponding $(0,0)$ peak and scaling appropriately for Franck-Condon factors. From such arrays of $\mathrm{P}_{\mathbf{2}}^{-}$and $\mathrm{O}_{\overline{2}}^{-}$"temperatures", we found that the population distributions were decidedly non-Boltzmann with the higher vibrational states of the ions exhibiting the higher "temperatures". We also found that the vibrational "temperatures" of these ions were strongly dependent on source conditions with higher relative source pressures correlating to lower vibrational 'temperatures". Using "temperatures" calculated from the ratios of $(0,1)$ to $(0,0)$ peak intensities, we obtained vibrational "temperatures" for $\mathrm{O}_{2}^{-}$ions ranging from $\approx 900$ to $1600 \mathrm{~K}$ as source pressure was varied and those for $\mathrm{P}_{2}^{-}$ions ranging with source pressure from $\approx 600$ to $1400 \mathrm{~K}$. Even though absolute source pressures were difficult to measure, these "temperatures" continued to increase as the source pressure was lowered still further. The "temperature", calculated by this procedure, for the $\mathbf{P}_{\mathbf{2}}^{-}$spectrum presented in fig. 2 was $\approx 700 \mathrm{~K}$. Its best fit "temperature" was nearer $600 \mathrm{~K}$.

\subsection{Electron affinity of $P_{2}$} from

The adiabatic electron affinity of $\mathbf{P}_{\mathbf{2}}$ was calculated

$$
\begin{aligned}
& \mathrm{EA}\left(\mathrm{P}_{2}\right)=\mathrm{EA}(\mathrm{P})+\gamma\left[\Omega\left(\mathrm{P}^{-}\right)-\Omega\left(\mathrm{P}_{2}^{-}\right)\right] \\
& \quad+m W\left[1 / M(\mathrm{P})-1 / M\left(\mathrm{P}_{2}\right)\right],
\end{aligned}
$$

where EA(P) is the electron affunity of phosphorus, $\gamma$ is the energy scale compression factor, $\Omega\left(P^{-}\right)$is the laboratory electron kinetic energy position for the $P$, ${ }^{4} S_{3 / 2} \leftarrow P^{-},{ }^{3} P_{2}$ transition, $\Omega\left(P_{2}^{-}\right)$is the laboratory electron energy for the $(0,0)$ transition in the $\mathrm{P}_{2}^{-}$spectrum (peak $C$ ), $m$ is the mass of an electron, $W$ is the beam energy, and $M(\mathrm{P})$ and $M\left(\mathrm{P}_{2}\right)$ are the respective masses of $P$ and $P_{2}$. Making these determinations in this manner relative to a calibrant jon with a known electron affinity compensates for any contact potential corrections, and the last term in this equation provides a kinematic correction from laboratory clectron energies to center-of-mass electron kinetic energies. The value of $\operatorname{EA}\left(P_{2}\right)$ determined from this equation was $0.586 \pm 0.009 \mathrm{eV}$ where the uncertainty is the experimental uncertainty. This value was then corrected for the cffects of hot-band pulling, rotational-state populations, and spin-orbit splitting in the ${ }^{2} \Pi_{g}$ state of $P_{2}-$. Transitions from excited vibrational states of $P-\frac{-}{2}$, which add asymmetrically to the height of peak $C$, shift its location by $3 \mathrm{meV}$, thus adding a correction of $-3 \mathrm{meV}$ to the electron affinity. To make a rotational correction we supposed that 
the rotational state distribution in the negative ion is at least somewhat approximated by a Boltzmann distribution, and that the rotational temperature of the ion is roughly equal to the vibrational temperature of $700 \mathrm{~K}$ mentioned earlier. For this rotational temperature the most prevalent rotational state in the negative ion should be $J^{\prime \prime}(\max )=29.1$. An approximate rotational energy correction is then given by [12]:

$\left(B_{\mathrm{e}}^{\prime \prime}-B_{\mathrm{e}}^{\prime}\right) J^{\prime \prime}(\max )\left[J^{\prime \prime}(\max )+1\right]$,

where $B_{\mathrm{e}}^{\prime \prime}$ and $B_{\mathrm{e}}^{\prime}$ are the rotational constants of the ground electronic states of $P_{\mathbf{2}}^{-}$and $P_{2}$, respectively. This correction is $-3 \mathrm{meV}$. The spin-orbit splitting correction for $\mathbf{P}_{2}^{-}$is more difficult to estimate. Modeling peak $C$ in our spectrum with several different magnitudes of this splitting showed that a spin-orbit splitting of $\approx 300 \mathrm{~cm}^{-1}$ would cause a visible peak asymmetry that we do not observe. Since the maximum shift in the peak location due to a spin-orbit splitting of between 100 and $300 \mathrm{~cm}^{-1}$ occurs in the middle of this range and is $\approx 9 \mathrm{meV}$, we have chosen to employ a spin-orbit correction of $+9 \mathrm{meV}$. The combination of all these corrections and uncertainties gives a final value for the adiabatic electron affinity of $\mathrm{P}_{2}$ of $0.589 \pm 0.025 \mathrm{eV}$. The positive electron affinities of $P$ and $P_{2}$ compared with the non-positive electron affinities of $\mathrm{N}$ and $\mathrm{N}_{2}$ emphasizes the often noted electronic differences between phosphorus and nitrogen [13].

\subsection{Bond dissociation energy of $P_{2}^{-}$}

Given the electron affinities of $P$ and $P_{2}$ and the dissociation energy of $P_{2}$, the dissociation energy of $\mathbf{P}_{\mathbf{2}}^{-}$can be calculated from a thermochemical cycle. Using $E A(P)=0.7464 \mathrm{eV}[8,14]$ and $D_{0}\left(\mathrm{P}_{2}\right)=5.033$ $\mathrm{eV}[1,15]$ we obtain $4.88 \pm 0.06 \mathrm{eV}$ for the dissociation energy of $\mathrm{P}_{2}^{-}$into the ground state of $\mathrm{P}^{\text {and }} \mathrm{P}^{-}$.

\section{Summary}

By applying negative ion photoelectron spectroscopy to the study of $\mathrm{P}_{2}^{-}$, we have determined the electron affinity of $P_{2}$ and the spectroscopic constants for $P_{2}^{-}$.
We find $\operatorname{EA}\left(\mathrm{P}_{2}\right)=0.589 \pm 0.025 \mathrm{eV}, D_{0}\left(\mathrm{P}_{2}^{-}\right)=4.88 \pm$ $0.06 \mathrm{eV}, \omega_{\mathrm{e}}^{\prime \prime}\left(\mathrm{P}_{2}^{-}\right)=640 \pm 50 \mathrm{~cm}^{-1}, B_{\mathrm{e}}^{\prime \prime}\left(\mathrm{P}_{2}^{-}\right)=0.2776$ $\pm 0.0028 \mathrm{~cm}^{-1}$ and $R_{\mathrm{e}}^{\prime \prime}\left(\mathrm{P}_{2}^{-}\right)=1.979 \pm 0.010 \AA$.

\section{Acknowledgement}

This research was supported in part by the Research Corporation and also in part by the National Science Foundation under Grant No. CHE-8511320. Acknowledgement is also made to the Donors of the Petroleum Research Fund, administered by the American Chemical Society, for partial support of this research.

\section{References}

[1] K.P. Huber and G. Herzberg, Molecular spectra and molecular structure. IV. Constants of diatomic molecules (Van Nostrand, Princeton, 1979).

[2] M. Dukel'skīi and E.Y. Zanberg, DokI. Akad. Nauk SSSR 86 (1952) 263.

[3] J.-D. Carette and L. Kerwin, Can. J. Phys. 39 (1961) 1300.

[4] S.L. Bennett, J.L. Margrave and J.L. Franklin, J. Chem. Phys. 61 (1974) 1647.

[5] D. Feldman, R. Rackwitz, H.J. Kaiser and E. Heinicke, Z. Naturforsch. A32 (1977) 600 .

[6] J.V. Coe, J.T. Snodgrass, C.B. Freidhoff, K.M. McHugh and K.H. Bowen, submitted for publication.

[7] E. Heinicke, K. Betłıge and H. Baumann, Nucl Instr. Methods 58 (1968) 125 .

[8] J. Slater and W.C. Lineberger, Phys. Rev. A 1:5 (1977) 2277.

[9] R.L.C. Wu and T.O. Tiernan, Bull. Am. Phys. Soc. 27 (1982) 109.

[10] L.L. Lohr, J. Phys. Chem. 88 (1984) 5569.

[11] H.J. Kaiser, E. Heinicke, H. Baumann and K. Bethge, $Z$. Physik 243 (1971) 46.

[12] R.J. Celotta, R.A. Bennett, J.L. Hall, M.W. Siegel and J. Levine, Phys. Rev. A6 (1972) 631.

[13] G. Herzberg, Molecular spectra and molecular structure. I. Spectra of diatomic molecules, 2nd Ed. C an Nostrand, Princeton, 1963) pp. 329-346.

[14] R.D. Mead, A.E. Stevens and W.C. Lineberger, in: Gas phase ion chemistry, Vol. 3, ed. M.T. Bowers (Academic Press, New York, 1984).

[15] J. Kordis and K.A. Gingerich, J. Chem. Phys. 58 (1973) 5141. 